\title{
MODELLING AND SIMULATION OF A RAILROAD WHEEL
}

\section{A. MURTAZA}

Professor ASET, Amity University, Lucknow Campus, Uttar Pradesh, India

ABSTRACT
This paper describes the analysis of a $25 T$ wheel, using Ansys. Full wheel model has been used. A model has
been verified by analytical computation of deflection under a transverse load. Then mechanical stresses under different
design locations as per AAR code have been simulated and results are comparable.
KEYWORDS: Railroad Wheel, Finite Element Analysis, Mechanical Loads \& AAR

Received: Jan 08, 2018; Accepted: Jan 29, 2018; Published: Mar 24, 2018; Paper Id.: IJMPERDAPR2018136

\section{INTRODUCTION}

A railroad car operates on a railway track, which guides it. Railroad wheel has evolved with the railroad transportation, which came into existence at the beginning of $19^{\text {th }}$ century. Its design is developmental which is based on its performance and maintenance requirements. In the beginning, wheels were built up type that is tired wheel center with or without spoke and now railroad wheels are monoblock. A material of wheel has changed with from medium carbon steel to high carbon steel with an alloying element and micro-cleaning process. Wise [1] has given the history of evolution and performance of railroad wheelsets. The wheel is most important for the safety and stability of a guided vehicle.

\section{RAILROAD WHEEL}

A typical wheelset is shown in figure 1. As shown in Figure 2, a railroad wheel consists of three parts, a hub, a rim and a web. Tread is the outer circumferential surface of the rim and its project part is called flange. Wheel tread comes into contact with the track and is directional. A railroad wheel not only supports the weight of a car but also the thermal and mechanical stresses caused during train operation

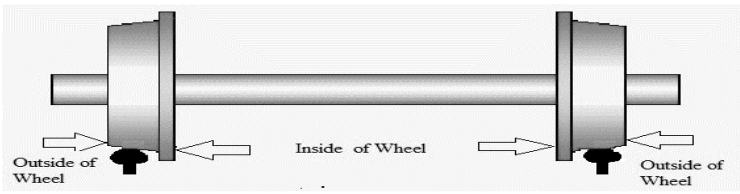

Figure 1: Rail Road Wheel Set on Rails

A railroad wheel [2] has to withstand the combined effects of stresses resulting from the following conditions:

- Thermal load due to brake application. These do not fluctuate during wheel revolution.

- Mechanical loads in the vertical direction (V) are due to equipment and lading of the railroad car. They apply at wheel tread and fluctuate once in each revolution. These may have dynamic augmentation due to track deviation and operation. 
- Mechanical loads in the lateral direction (L) apply at the tread or the flange during curve negotiation or vehicle stability such as hunting and nosing. These loads also fluctuate during wheel revolution.

- Driving wheels exert tractive load at the rail-wheel contact area. These also fluctuate.

\section{Centrifugal forces}

Centrifugal forces are generated when the axis of revolution is rotated in space either due to the passage around a curve or passage over irregularities. AAR [3] have also ignored the gyroscopic effect on the wheel in their work. Further, these gyroscopic forces are very minimal and are not usually considered in rail wheel design as per KasyAiyar [4].

\subsection{Kinds of Mechanical Loads}

Figure 2 shows the mechanical loads exerted on a railroad wheel. These loads at a rail-wheel interface are also considered for the stability analysis of a railroad vehicle [4].

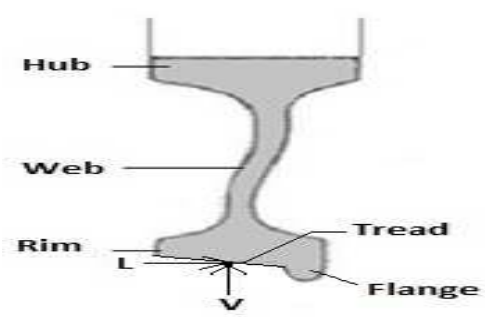

Figure 2: Wheel Disc of a Rail Road Wheel

\subsection{Mechanical Stresses at the Tread}

A new wheel of $916 \mathrm{~mm}$ diameter with standard contour supporting a $100 \mathrm{k} \mathrm{N}$ load on a new rail produces a calculated area of contact of about $159 \mathrm{~mm}^{2}$ [5]. This results in a concentration of very high stress which is cyclic and gives ideal conditions for fatigue failure.

A serious effect of mechanical stress at the tread is shelling. This is a fatigue failure due to contact stresses and is to be found on wheels having high static or dynamic loads. The magnitude of this effect is directly related to the geometry of the wheel and rail, the vertical wheel/rail forces and speed.

\section{LITERATURE REVIEW}

Rusin el at [6] used finite element method to compare various wheel designs of AAR. They considered mechanical and thermal loads on the wheel and used experimental data for verification. Brunel el at [7] used a computer program developed by General electric company to simulate service braking and loading conditions. They simulated various braking conditions and track loads in order to compare various wheel designs of AAR. They concluded that there was no optimum design for all combinations of braking and loading conditions.

Sokamoto el at [8] has used fracture mechanics to assess the efficacy of various wheel design including wheel offset.

Extensive work done at the Chalmers University [9] has resulted in a finite element model, which considers the axle as an integral part of the wheel. The model relies on the geometric symmetry of the wheel and axle and hence takes into account only a quarter of the assembly. 
Symmetry boundary conditions are applied in the 1-2 and 2-3 planes of the axle in order to account for both the left and right wheels. The vertical and lateral loads are applied at the gauge point similar to the AAR method. However, the reactions in the 2 directions are received at the bearing location. Ramanna el at [11] have considered wheelaxle in contact with the wheel and compared their results with Chalmers university [9] model and AAR code[3].

Finite element analysis [ 10] was done using NISA FE analysis software. The loading considered was as per AAR specification [3]. In this analysis, half wheel model was used for mechanical loading. In this analysis, A total of 11818 nodes and 9702 3D brick elements were used to represent the model. All the nodes in the hub bore are fixed in the radial and axial directions. In addition, symmetry boundary conditions are enforced since only half the wheel is considered.

\section{PRESENT WORK}

This simulation has been done for mechanical loads to verify the model and compare the results with RWF analysis.

IR's $25 \mathrm{~T}$ axle load wheel has been modeled using design modeler of Ansys Workbench 18.2 Academic. A sketch of wheel section as per figure 3 was drawn and then full wheel was extruded.

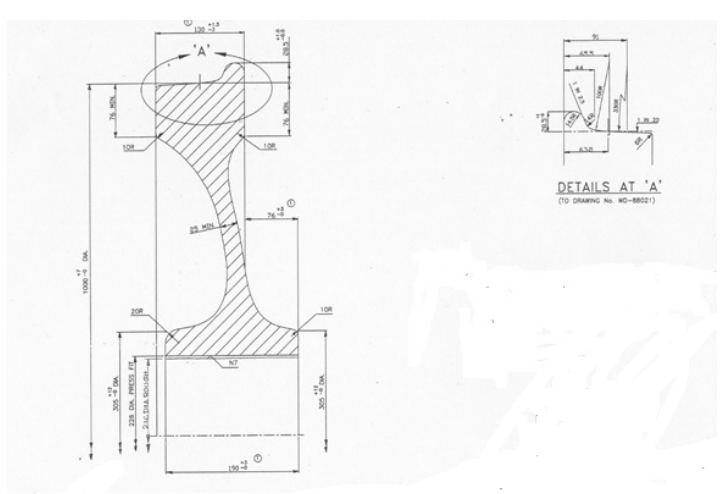

Figure 3: Section of 25T Railroad Wheel

Once this model was ready, deflection of the plate was considered to verify the model. In this analysis, 11649 elements and 21200 have been used.

\subsection{Verification of Model}

Strozza [12] analyzed the deflection of a clamped annular plate subjected to the transverse load.

Deflection is related to flexural rigidity of plate. Figure 3 [12], gives a variation of w/k with the ratio of radii.

The $25 \mathrm{~T}$ wheel plate is considered as an annular plate with inner diameter 112 and outer diameter424. In this case radii ratio is 0.358 . In this analysis, plate is clamped at inner radii.

Corresponding to this ratio figure 3 [12] gives a value 0.6 of $\mathrm{w} / \mathrm{k}$.

Value of $\mathrm{k}$ has been computed for a lateral load of $12.5 \mathrm{~T}$.

A wheel was considered as an annular plate under transverse load. In order to compute flexural rigidity wheel section (Figure 3) was modeled as rectangular sections as shown in Figure 4, without changing radial dimensions (Figure $3)$. 
- $\quad$ Rim section $130 \times 76$

- $\quad$ Plate section $42 \times 312$

- Hub section $195 \times 35$

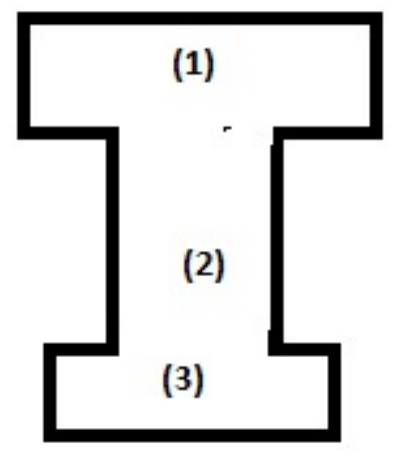

Figure 4: Modelling of Wheel Section for Computing Flexural Rigidity

Flexural rigidity of plate section [13] is $13569.23 \mathrm{e}+5$, which gives $\mathrm{k}=2.585$ for a $12.5 \mathrm{~T}$ load.

An expression \{ equation 7 of [9] $\}$ for interpolating the curve $\{$ Figure 3 of [12] $\}$ showing variation of w/k with radii ratio of annular plate for $0.04<=\mathrm{Ri} / \mathrm{Re}>=0.6$ is

$\mathrm{w} / \mathrm{k}=2.706 .\left[1-\left\{1-(1-\mathrm{Ri} / \mathrm{Re})^{2}\right\}^{0.5}\right]$

Above equation is based on the curve of best fit and has a maximum error of $7 \%$. Corresponding to a radii ratio of 0.358 , this equation gives $\mathrm{w} / \mathrm{k}=0.8736$, which gives a deflection of $\mathrm{w}=2.2589 \mathrm{~mm}$.

Above mentioned computations have been done by using Python Spyder.

\subsection{Mechanical Loading of Wheel Model}

The following loads as defined in [3] were applied:

$\begin{array}{lll}\text { Vertical Load V1 } & : & 25,000 \mathrm{~kg} \\ \text { Lateral Load L1 } & : & 12,500 \mathrm{~kg} \\ \text { Vertical Load V2 } & : & 25,000 \mathrm{~kg}\end{array}$

Von-Mises stresses were calculated for the following load combinations [3]:

(i) $\mathrm{V} 1+\mathrm{L} 1$

(ii) $\quad$ V2

\section{Material Properties:}

$\begin{array}{lll}\text { Modulus of Elasticity } & : & \mathrm{E}=2 \mathrm{E}+11 \mathrm{~N} / \mathrm{m}^{2} \\ \text { Poisson's Ratio } & : & \mu=0.30 \\ \text { Density } & : & \rho=7833 \mathrm{~kg} / \mathrm{m}^{3}\end{array}$


Loading, for mechanical stresses [3], has been done as per following figure 5.

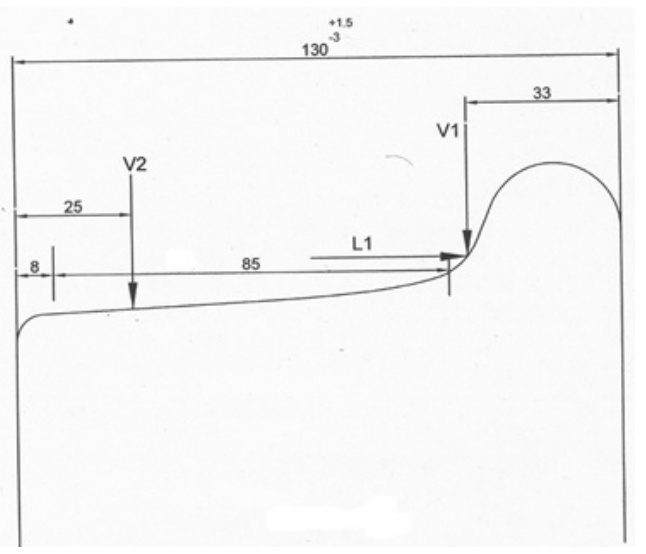

Figure 5: Loading Locations as per [3]

\section{SIMULATION AND DISCUSSION OF RESULTS}

\subsection{Verification of Simulation Model}

Figure 6 shows the deflection of the wheel under a transverse load of 12.5T. The maximum value of deflection on a plate is $2.54 \mathrm{~mm}$ and that obtained by analytical method is $2.2589 \mathrm{~mm}$. These are comparable.

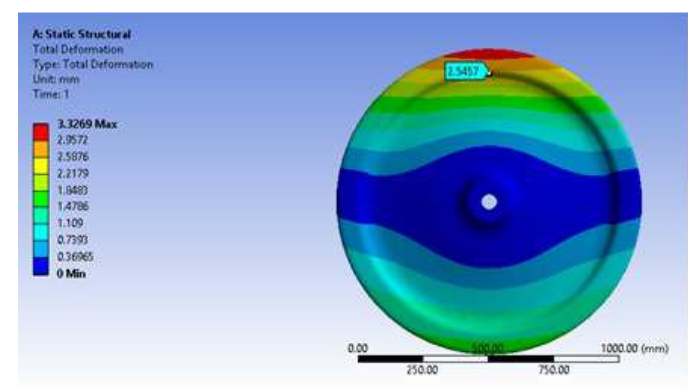

Figure 6: Deformation of Wheel Under a Transverse Load of 12.5T

\subsection{Mechanical Stresses}

Figure 7 and 8 show von-mises stresses in the $25 \mathrm{~T}$ wheel.

\subsubsection{LoadV2 at $25 \mathrm{~mm}$ location as per loading figure 5}

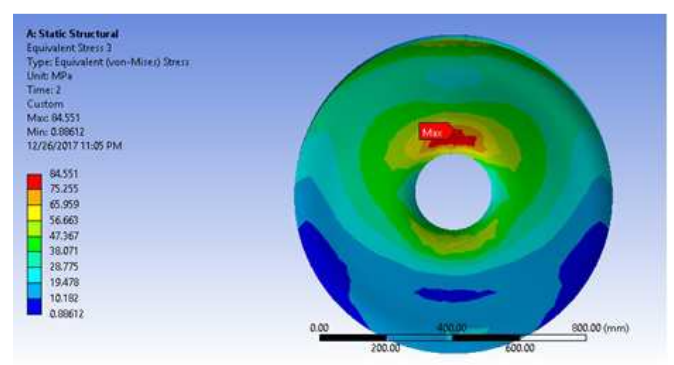

Figure 7: Von-Mises Stresses due to Vertical Load V2 at Tread 
Figure 7 shows the variation of Von-Mises stresses due to a vertical load of 25T at $25 \mathrm{~mm}$ location as per loading Figure 5. Von-Mises vary from 0.88 MPa to 84.51MPa. It is evident from stress distribution that maximum stresses occur at plate hub interface is 84.51 . The maximum value of Von-Mises stress in plate area is per [10 ] is 83.7

\subsubsection{V1 and L1 at 33 (Figure 5)}

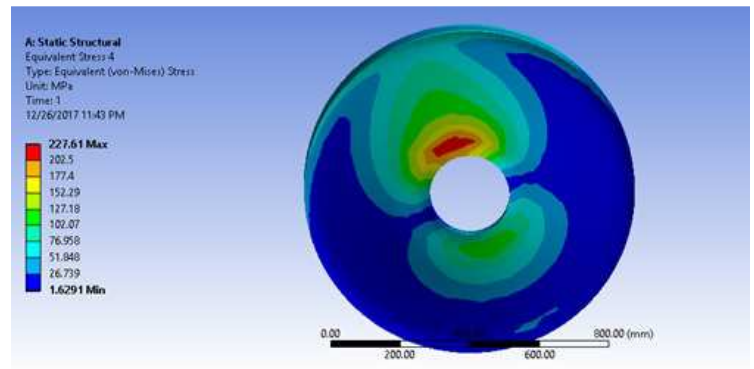

Figure 8: Stresses due to Combined Vertical(V1) and Lateral (L1) Loadsat Tread

Figure 8 shows the variation of Von-Mises stresses under a vertical load of 25T and a lateral force of 12.5T as per loading is shown in Figure 5. Von-Mises stress vary from 1.62 MPa to 227.61 MPa with a maximum value of 227.61 Mpa in plate area. Here also maximum stress locations are at plate-hub transitions. As per [10 ] is 172.3

Table 1: Von-Mises Stress and Deformation for wheel

\begin{tabular}{|l|c|c|c|c|c|}
\hline Region & Loads & $\begin{array}{c}\text { Max. Von-Mises } \\
\text { stress (MPa) } \\
{[\mathbf{1 0}]}\end{array}$ & $\begin{array}{c}\text { Max. Von- } \\
\text { Mises stress } \\
\text { (MPa) }\end{array}$ & $\begin{array}{c}\text { Deformation } \\
\text { mm(Analytically) }\end{array}$ & $\begin{array}{c}\text { Deformation } \\
\text { Mm(Model) }\end{array}$ \\
\hline Plate & V2 & 83.7 & 84.51 & & \\
\hline Plate & V1+L1 & 172.3 & 227.61 & & \\
\hline Plate & L1 & - & - & 2.25 & 2.54 \\
\hline
\end{tabular}

\section{CONCLUSIONS}

- In this study full wheel has been modeled for finite element analysis using Ansys Workbench. First of all this model has been verified for deflection simulated and that computed by treating wheel as an annular plate. The deflection obtained by simulation was $2.54 \mathrm{~mm}$. And that by analysis was 2,25 . These are comparable.

- Table-1 gives the Von-Mises stresses obtained from fe analysis. For a vertical load of 25T at $25 \mathrm{~mm}$, it was 84.51 and that obtained by RWF half wheel model was $83.7 \mathrm{Ma}$. These are comparable. For loading V1+L1 at $33 \mathrm{~mm}$, it was 227.61. And that obtained by RWF half wheel model it 172.3 MPaThese are comparable.

- The present full wheel model gives values of stresses slightly higher. This work can be further extended for thermal loadings also.

\section{ACKNOWLEDGEMENT}

The author acknowledges with thanks the assistance provided by Mr. Ashwani Sharma AP/ Amity University, Mr. VivekVerma AP/ Amity University, and Mr. M. I. Murtaza Member/ASME. 


\section{REFERENCES}

1. S. Wise (1987) Railway Wheelsets-a Critical Review. Proceedings of the I. Mech. E. Part D: Journal of Automobile Engineering 201(4) 257-271.

2. K. Hirakawa, H. Sakamoto (1981) Effect of Design Variation on Railroad Wheel Fracture. ASME paper 91-WA/RT-41.

3. Procedure for analytic evaluation of locomotive and freight car wheel designs, Association of American Railroads Standard S660-83, adopted 1981, Revised 2004.

4. S. Jebarose Juliyana et al.,, Finite Element Analysis of Mono Composite Leaf Spring of Varying Thickness and Varying Width used in Automotives, International Journal of Mechanical and Production Engineering Research and Development (IJMPERD), Volume 7, Issue 6, November - December 2017, pp. 247-254

5. KasyAiyar (1976) Some Aspects of the Problem of Rail-Wheel Interaction. Research Design and Standards Organisation, India, Technical Report No.345, 1976:1.

6. M. Gay (1979) The modern Wheelset. International Conference on Railway Braking, SP21 26-27 Sep. 1979. (Sponsored by Railway division of I.Mech.E.).

7. T. M. Rusin, D. G. Kleeshulte, J. M. Coughlin, 'Application of finite element method in the development of improved railroad car Wheel Designs', Transactions of the ASME 378/ Vol. 101, August 1979.

8. J. P. Bruner, R. D. Jones, Samuel Levy, J. M. Wandrisco (1968) Effect of Design Variation on Service Stresses in Railroad Wheels. ASME 187-196., Paper No. 67- WA/RR-6.

9. H. Sakamoto, K. Hirakawa, 'Prevention of Rail road wheel fracture', JSME International Journal, Vol.46, No.4, 2003, pp 613619.

10. Report of railway engineering research at Chalmers University in Gothenburg. Proceedings of the Institute of Mechanical Engineers 1992;206:145.

11. Finite Element Analysis Report of $1000 \mathrm{~mm}$ dia $25 T$ axle load wheel for Wagons, Technology Centre, Rail Wheel Factory, Bangalore, India.

12. L. Ramanan, R. Krishna Kumar, R. Sriraman,'Thermo-mechanical finite element analysis os a rail wheel', International Journal of Mechanical Sciences, 41 (1999) 487-505.

13. P. Strozzi, 'Mechanical Analysis of an annular plate subjected to transverse concentrated load' Journal of Strain Analysis, Vol. 24, No. 3, 1989, pp 139-149.

14. De, S. Basuli, 'Axisymmetric bending of uniformly stressed annular plates with variableRigidity', Journal of the Indian Institute of Science, 2013 - journal.iisc.ernet.in, pp 119-128.

\section{Notations}

AARAssociation of American Railroads

IRIndian Railways

RWFRail Wheel Factory (Erstwhile Wheel and Axle Plant) Bangalore India

P Transverse Concentrated load

Re Outer radius of annular plate 
RiInner radius of annular plate

D Plate flexural rigidity

W Plate transverse deflection

hthickness of plate model

$\mathrm{K}=(\mathrm{P} . \operatorname{Re} 2) /(2 . \pi . \mathrm{D})$

$\mathrm{D}=\mathrm{E} . \mathrm{h} 3 /\{12(1-\mu 2)\}$ 